\title{
Vascularized silk electrospun fiber for promoting oral mucosa regeneration
}

\author{
Chunyu Qian ${ }^{1,2}$, Tianwen Xin ${ }^{3}$, Wanshu Xiao ${ }^{1}$, Huajun Zhu', Qiang Zhang ${ }^{4}$, Lili Liu², Ruoyu Cheng ${ }^{5}$, Zhen Wang ${ }^{5}$, \\ Wenguo Cui $\mathbb{B}^{3,5}$ and Zili Ge ${ }^{1}$
}

\begin{abstract}
Electrospun fiber membranes have been extensively researched for tissue repair; however, fiber angiogenesis has been a difficult problem because the biomaterials used to prepare the fiber cannot promote angiogenesis by themselves. To endow the fiber with the function of promoting angiogenesis and oral mucosa regeneration, surface-aminated liposomes ( $\mathrm{NH}_{2}$-LIPs) encapsulating leptin, as well as silk fiber (SF) membranes that have been immersed overnight in polydopamine (PDA) solution were synthesized. The $\mathrm{NH}_{2}$-LIPs were then grafted onto the surfaces of SF through reactions between the catechol groups of PDA and the amino groups of $\mathrm{NH}_{2}$-LIPs to induce angiogenesis on the fiber surface and thus promote oral mucosa regeneration. The PDA-modified and $\mathrm{NH}_{2}$-LIP-modified SF retained the original fibrous morphology but increased the SF tensile strength from 1.95 MPa to 2.87 MPa. PDA changed the hydrophilicity of the fibers and improved the adhesion of fibroblasts on the fibrous membranes. Leptin loaded onto SF via PDA had no significant effect on cell proliferation and formed 162.7-node tubes in human umbilical vein endothelial cells (HUVECs) within $10 \mathrm{~h}$, which demonstrated that leptin loaded onto SF can promote angiogenesis. Wound closure of mucosa covered in leptin-loaded fibrous membranes reached $99 \%$ at 14 days postsurgery. Histologic analysis showed that leptin-loaded SFs exhibited clear stratification of new mucosa, as well as a strong CD34 signal that indicated the presence of new blood vessels and confirmed the successful loading of leptin on the fiber. Therefore, this work successfully showed that $\mathrm{NH}_{2}$-LIPs grafted onto the surface of SFs via PDA endow the fibers with angiogenic abilities and promote oral mucosa regeneration.
\end{abstract}

\section{Introduction}

Angiogenesis is a highly complex process that plays a vital role in the normal development of the human body and the pathophysiology of various diseases ${ }^{1-3}$. During the repair of defects in tissues such as bone ${ }^{4}$, muscle ${ }^{5}$, nerves ${ }^{6}$, and $\operatorname{skin}^{7}$, newly formed blood vessels improve microcirculation within the wound, provide nutrition for tissue regeneration, and promote injury healing ${ }^{8}$. Early transplantation of vascular tissue helps restore tissue morphology and reduce scar contraction for large areas

Correspondence: Wenguo Cui (wgcui80@hotmail.com) or Zili Ge (gezl@163. com)

'Department of Stomatology, The First Affiliated Hospital of Soochow University, 899 Pinghai Road, 215006 Suzhou, Jiangsu, China

${ }^{2}$ Department of Orthopaedic Surgery, The First Affiliated Hospital of Soochow University, 899 Pinghai Road, 215006 Suzhou, Jiangsu, China

Full list of author information is available at the end of the article with severe oral mucosa damage ${ }^{9}$. The promotion of wound angiogenesis is therefore an important part of the recovery of normal oral damaged mucosa morphology and function. Currently, through developments in tissue engineering, a variety of material scaffolds are available and widely used for tissue regeneration due to their biomimetic properties ${ }^{10,11}$. However, although material scaffolds can help guide cell behavior ${ }^{12,13}$, simple scaffolds cannot promote the angiogenesis required for tissue repair, resulting in a loss of blood supply to the wound and making it difficult to achieve complete reconstruction of the shape and function of the damaged tissue ${ }^{14}$. Accordingly, it is necessary to find a biomaterial that can both guide cell growth and promote local vascularization to accelerate recovery from severe oral mucosal injuries.

\section{(-) The Author(s) 2020}

(c) (i) Open Access This article is licensed under a Creative Commons Attribution 4.0 International License, which permits use, sharing, adaptation, distribution and reproduction c. in any medium or format, as long as you give appropriate credit to the original author(s) and the source, provide a link to the Creative Commons license, and indicate if changes were made. The images or other third party material in this article are included in the article's Creative Commons license, unless indicated otherwise in a credit line to the material. If material is not included in the article's Creative Commons license and your intended use is not permitted by statutory regulation or exceeds the permitted use, you will need to obtain permission directly from the copyright holder. To view a copy of this license, visit http://creativecommons.org/licenses/by/4.0/. 
Fibrous membranes can be made by electrospinning, an easy-to-operate and low-cost technology, and they have highly specific surface areas and three-dimensional micronanostructures ${ }^{15-17}$. The resulting membranes are similar in structure to the extracellular matrix (ECM) and are widely used in tissue regeneration ${ }^{18-20}$. Electrospun fiber membranes are suitable for application in the oral cavity because their thickness and size can be firmly controlled ${ }^{19}$. In addition, the porous structure of fiber membranes facilitates the supply of oxygen and nutrients to the wound ${ }^{21}$. However, the surface of the fiber membrane is difficult to vascularize due to a lack of factors for angiogenesis induction. Therefore, electrospun fibers can load bioactive factors to promote angiogenesis ${ }^{22}$. Leptin is an angiogenic factor that is transcribed from the leptin gene (OB gene) on human chromosome 7 (7q31,3), which produces a 167 amino acid peptide with high hydrophilicity ${ }^{23-25}$. Leptin promotes angiogenesis during tumor formation by stimulating the high expression of angiogenic factors through pathways including STAT3 and MAPK ${ }^{23,26}$. The loading of leptin into electrospun fiber membranes is thus expected to solve the problem of fiber membrane angiogenesis. Currently, when producing fibers for subsequent drug loading, the types of electrospinning used are blend electrospinning ${ }^{27,28}$, coaxial electrospinning $^{29,30}$, emulsion electrospinning ${ }^{31,32}$, and microsol electrospinning ${ }^{33}$. After the fibers are produced, drugs are loaded onto them by any of the following three methods: physical adsorption, layer-by-layer assembly, and chemical fixation ${ }^{34}$. However, the organic solvent applied in blend electrospinning and coaxial electrospinning destroys the structure of active angiogenic factors and inactivates them ${ }^{35}$. In addition, the drug encapsulated inside the fiber is released slowly, is deposited near the fiber, and is inaccessible to deep tissue $^{36}$. There is thus an urgent need for electrospun fiber membranes capable of being loaded with leptin but without the above disadvantages in order to enable vascularization of the fiber membrane.

Silk fibroin is a natural biomaterial derived from Bombyx mori silk ${ }^{13}$ and has good cytocompatibility, excellent mechanical properties, and oxygen permeability ${ }^{37-39}$. Silk fibroin promotes the growth and proliferation of fibroblasts and epithelial cells, thus meeting the requirements of oral mucosal tissue engineering scaffolds ${ }^{37,40}$. However, the solution for electrospinning silk fibroin is prepared by dissolving silk fibroin in hexafluoroisopropanol (HFIP) ${ }^{13}$ or formic acid ${ }^{41-43}$, and the biological activity of leptin is destroyed when directly mixed with HFIP $^{41}$. As a result, it is difficult to create silk fibers (SFs) loaded with watersoluble biologically active drugs. Liposomes (LIPs) are closed bilayer vesicles formed by phospholipid bilayers and can carry multiple types of drugs. Hydrophilic factors, such as proteins, antibodies, and cytokines, are preserved in the internal structure of LIPs ${ }^{44,45}$. Therefore, LIPs can be used to protect the biological activity of leptin through encapsulation. The use of PDA was inspired by mussel foot protein 5 (Mefp-5), which is rich in 3,4-dihydroxy-Lphenylalanine (DOPA) and lysine. PDA reacts with nucleophilic functional groups, such as amino groups and thiols, through its catechol groups ${ }^{35,46}$. PDA can therefore be added to the surface of SF membranes and then allowed to react with the amino groups on aminated liposomes $\left(\mathrm{NH}_{2}\right.$-LIPs) to attach leptin onto fiber surfaces, thereby loading drug-encased $\mathrm{NH}_{2}$-LIPs onto the fiber surfaces under mild reaction conditions.

In this study, to induce the ability of the fibrous membrane to promote angiogenesis in oral mucosa regeneration, we utilized PDA as a link to graft $\mathrm{NH}_{2}$-LIPs onto the surface of SF. In this way, we can construct an electrospun fibrous membrane that can locally release leptin to stimulate blood vessel regeneration and promote healing of damaged oral mucosa. First, preparation methods of $\mathrm{NH}_{2}-$ LIPs and the physicochemical properties of the fiber membranes were characterized. Second, the cytotoxicity and angiogenic ability of the fibrous membrane were investigated in vitro. Finally, large area defects of rabbit oral mucous membranes were covered in the fibrous membranes to evaluate the ability of these fibrous membranes to facilitate wound healing.

\section{Materials and methods \\ Preparation of fiber membranes \\ Preparation of SF electrospinning solution}

A total of $5 \mathrm{~g}$ of silk obtained from cocoons of Bombyx mori (Jiangsu Huajia Silk Co., Ltd. Soochow) was boiled in $3 \mathrm{~L}$ of $\mathrm{Na}_{2} \mathrm{CO}_{3}$ (1.41 g/L Aladdin Biotechnology Company, Shanghai) aqueous solution for $40 \mathrm{~min}$ and repeatedly scrubbed with deionized water to remove sericin. The degummed silk was then dissolved in $9.3 \mathrm{M} \mathrm{LiBr}$ (Aladdin Biotechnology Company) aqueous solution at $60^{\circ} \mathrm{C}$ and dialyzed in deionized water (dialysis bag, $\mathrm{Mw} 3500$, Shanghai Source Leaf Biotechnology Co., Ltd. Shanghai) for 3 days. Next, polyethylene glycol (PEG, Mw 20000, Meilun Biotechnology Co., Ltd. Shanghai) was utilized to concentrate and purify the solution to produce an $8.0 \% \mathrm{w} /$ $\mathrm{v}$ silk fibroin solution. The resulting silk fibroin solution was centrifuged $\left(5000 \mathrm{rpm}, 1 \mathrm{~h}, 4^{\circ} \mathrm{C}\right)$ and freeze-dried into silk fibroin powder. Next, $0.7 \mathrm{~g}$ of silk fibroin powder was dissolved in 1,1,1,3,3-hexafluoro-2-propanol (HFIP, $9.3 \mathrm{~g}$ solution, Shanghai Mairell Chemical Technology Co., Ltd. Shanghai). The solution was placed in a magnetic agitator (Shanghai Ssler Instruments Co., Ltd. Shanghai) and stirred until the solution became transparent in order to obtain a 7\% SF electrospinning solution.

\section{Preparation of SF membrane}

The transparent SF electrospinning solution was added to a $10 \mathrm{~mL}$ syringe equipped with a steel needle with an inner diameter of $0.9 \mathrm{~mm}$. The syringe was fixed to a 
precision pump (Baoding Langern Constant Current Pump Co., Ltd. Baoding) with a spray rate of $0.08 \mathrm{~mL} / \mathrm{h}$. The needle of the syringe was connected to a high-voltage power supply (Tianjin Dongwen High Voltage Power Supply Co., Ltd. Tianjin), and the voltage was set at $12 \mathrm{kV}$. A cylindrical aluminum foil receiver was also connected to a high-voltage power supply and placed $10 \mathrm{~cm}$ from the needle tip in order to collect the fibers. The prepared SF was treated with methanol (National Pharmaceutical Group Chemical Reagent Co., Ltd. Shanghai) to induce $\beta$-folding and make the fiber insoluble in water. The fiber membrane was then dried in vacuum at $23^{\circ} \mathrm{C}$ for 2 days to completely remove the solvent residues on the SF membrane $e^{47,48}$.

\section{Preparation of $\mathrm{NH}_{2}$-LIP-wrapped leptin}

Surface-aminated liposomes $\left(\mathrm{NH}_{2}\right.$-LIPs) encapsulating leptin were prepared using a thin film dispersion method. Soft soy phospholipids (Shanghai Yuanye Biotechnology Co., Ltd. Shanghai), cholesterol (Acros, Shanghai), DEPEPEG- $\mathrm{NH}_{2}$ (Aladdin Biotechnology), and octadecylamine (Aladdin Biotechnology) were dissolved in chloroform at a $\mathrm{w} / \mathrm{w}$ ratio of 40:10:2:2. The solution was then placed in a round-bottom flask and treated with a rotary evaporator (Shanghai Yarong Biochemical Instrument Factory, Shanghai) at $60^{\circ} \mathrm{C}$ and a rate of 50 rpmto form a film, which was then hydrated with an aqueous leptin solution $(10 \mathrm{mg} / \mathrm{mL})$ at $25^{\circ} \mathrm{C}, 50 \mathrm{rpm}$. The resulting solution was sonicated and purified by filtration through 450 and $220 \mathrm{~nm}$ filters to prepare $30 \mu \mathrm{g} / \mathrm{mL} \mathrm{NH}_{2}$-LIPs.

\section{Loading of fiber membranes with leptin}

SF membranes were immersed in $2 \mathrm{mg} / \mathrm{mL}$ PDAin $1 \mathrm{mM}$ Tris- $\mathrm{HCl}$ buffer, $\mathrm{pH} 8.5$ (PDA, Alfa Aesar, America), for $12 \mathrm{~h}$ and repeatedly rinsed with deionized water to deposit PDA on the SF (SF@PDA). The SF@PDA membranes were then immersed in a solution of leptinencapsulated $\mathrm{NH}_{2}$-LIPs overnight at $4^{\circ} \mathrm{C}$ to produce leptin-loaded SF membranes (SF@PDA@LIP).

\section{Characterization of the physicochemical properties of $\mathrm{NH}_{2}^{-}$ LIPs and fiber membranes}

$\mathrm{NH}_{2}$-LIPs were loaded into colorimetric cuvettes $(1 \times$ $1 \times 5 \mathrm{~cm}^{3}$ ), and the average particle size, potential, and dispersion of the $\mathrm{NH}_{2}$-LIPs were measured using a dynamic light scattering particle size dispersion instrument (DLS, Zetasizer, Malvern, Nano-ZS90, Britain). The morphology of $\mathrm{NH}_{2}$-LIPs was examined using transmission electron microscopy (TEM, Tecnai G-20, America), while the surface morphology of fibers was evaluated with scanning electron microscopy (SEM, QUANTA, Japan) and atomic force microscopy (AFM, Bruker, Germany). The fiber diameter distribution was analyzed with ImageJ software. The surface roughness of the fibers was examined by analyzing AFM images with NanoScope software. Following the $\mathrm{NH}_{2}$-LIP preparation method described above, $\mathrm{NH}_{2}$-LIPs were labeled with coumarin (Sigma-Aldrich Technology Co., Ltd. Shanghai), and the distribution of $\mathrm{NH}_{2}$-LIPs on the fibers was examined by fluorescence microscopy (Zeiss, Germany). The surface chemical composition and changes in the surface elements of the fiber membranes were analyzed by X-ray photoelectron spectroscopy (XPS, 250Xi, Thermo Scientific Escalab, America). Fiber membranes were cut into strips $\left(15.0 \times 3.0 \times 0.13 \mathrm{~mm}^{3}\right)$ and stretched with a biomechanical tester (Shanghai Hengyi Precision Instrument Co., Ltd. Shanghai) to measure the mechanical properties of the fiber membranes. The hydrophilicity of the fiber membranes was examined by water contact angle (WCA, Data Physics, Germany) measurements.

\section{Leptin release in vitro}

Leptin-loaded SF membranes were immersed in PBS and shaken at $37^{\circ} \mathrm{C}$ in a desktop isothermal oscillator (Taicang Huamei Biochemical Instrument Factory, Soochow). At the same time, $\mathrm{NH}_{2}$-LIPs not grafted onto $\mathrm{SF}$ membranes were loaded into dialysis bags $(\mathrm{Mw}=$ $100 \mathrm{kDa}$, Shanghai Yuanye Biotechnology Co., Ltd.) and submerged in PBS. Next, $1 \mathrm{~mL}$ of PBS was taken at specific time points and supplemented with $1 \mathrm{~mL}$ of fresh PBS each time to ensure sufficient dialysate. An ELISA kit was used to determine the concentration of leptin in the PBS solution at different time points, and the release rates of each type of liposome were calculated.

\section{Cell proliferation and adhesion}

Rat fibroblasts were seeded onto fibrous membranes and cultured with DMEM high L-glucose (HyClone, American Gibco Company, America) for 1 day, 3 days, and 5 days. Cell dehydrogenation was measured using a cell counting kit (CCK8), and the number of living cells was indirectly determined. After culturing on fibrous membranes for 3 days, fibroblasts were fixed with $4 \%$ paraformaldehyde, and the cytoskeleton and nucleus were labeled with Alexa Fluor 594 phalloidin and DAPI, respectively. In addition, $\alpha$-smooth muscle actin ( $\alpha$-SMA) and integrin in the cells were labeled using anti-mouse $\lg G$ and anti-rabbit $\lg G$, respectively, and then observed with a fluorescence microscope to detect the state and adhesion of the cells on the fibrous membranes.

\section{Tube formation assay}

To evaluate the effects of leptin release from the fibrous membranes on angiogenesis, tube formation was induced by growth factor-reduced Matrigel $(100 \mu \mathrm{L} /$ well $)$ in a 24-well plate. Next, $6 \times 104$ human umbilical vein endothelial cells (HUVECs) were seeded into a $0.4 \mu \mathrm{m}$ Transwell with SF, SF@PDA and SF@PDA@LIP in the upper compartment, 
incubated in DMEM with high L-glucose for either 5 or $10 \mathrm{~h}$ and then fixed with $4 \%$ paraformaldehyde. The cytoskeleton was then labeled with Alexa Fluor 594 phalloidin and observed using a laser scanning confocal microscope (LSM 800, Zeiss, Germany).

\section{In vivo experiments}

New Zealand rabbits were purchased at the Experimental Animal Center of Suzhou University (Suzhou, China). The animal experiment was approved by the Experimental Animal Welfare and Ethics Committee of Soochow University. Forty-eight rabbits were randomly divided into 4 groups and anesthetized with $10 \% \mathrm{w} / \mathrm{v}$ chloral hydrate at dosages of $7 \mathrm{ml} / \mathrm{kg}$. Full-thickness soft tissue defects with diameters of $1 \mathrm{~cm}$ were created in the oral mucosal regions and covered in either gauze (for the control group) or SF, SF@PDA, or SF@PDA@LIP membranes for the remaining three groups. At 3, 5, 7, and 14 days postoperation, 3 rabbits in each group were randomly selected and killed to observe the overall condition of the rabbits and oral mucosa wound recovery. Wound tissues were fixed with formaldehyde, and paraffin-embedded sections were stained with hematoxylin-eosin (H\&E) and Masson's trichrome to observe the state of defect repair and collagen formation, respectively. CD34 expression was detected via labeling with HRP-labeled goat anti-rabbit universal secondary antibody (Wuhan Servicebio Biotechnology Co., Ltd.). The number of CD34-positive blood vessels was calculated using Image-Pro Plus software (Media Cybernetics), and in five randomly selected regions, positively stained microvessels were counted.

\section{Statistical analysis}

Experiments were performed in triplicate unless otherwise stated. Data are expressed as the mean \pm standard deviation. Statistical analysis to evaluate differences between groups consisted of an analysis of variance (ANOVA) followed by Tukey's multiple comparison tests and were performed using GraphPad Software. $P<0.05$ was considered statistically significant.

\section{Results and discussion}

$\mathrm{NH}_{2}$-LIPs encapsulating leptin were fabricated by thin film dispersion of DSPE-PEG- $\mathrm{NH}_{2}$, lecithin and cholesterol (Scheme 1a). Silk fibroin was prepared as SF by electrospinning. To introduce catechol groups on the SF surfaces, PDA was deposited on the surface of the membrane through oxidative self-polymerization of PDA. Next, SF@PDA was grafted to $\mathrm{NH}_{2}$-LIPs through covalent crosslinking between the catechol groups and amino groups under alkaline conditions, producing SF@PDA@LIP (Scheme 1b). The rupture of $\mathrm{NH}_{2}$-LIPs grafted on the surface of SF@PDA releases leptin into mucosal defects to enhance vascular regeneration and improve the recovery rate of defect-containing oral mucosa (Scheme 1c).

Analysis using DLS showed that the zeta potentials of ordinary LIPs and $\mathrm{NH}_{2}$-LIPs were $44.94 \mathrm{mV}$ and $40.11 \mathrm{mV}$, respectively, and were very close. Upon amination, the average particle size of LIPs changed from $273.1 \mathrm{~nm}$ to $224.4 \mathrm{~nm}$, and the PDI values were 0.242 and 0.241 , respectively, indicating that amination did not change the LIP size and that the LIPs have good dispersibility both before and after amination. The internal morphology of liposomes was observed using TEM. Both types of LIPs were uniform, smooth, and spherical (Fig. 1a).

SEM images (Fig. 1b) demonstrated that the surfaces of SF and SF@PDA were smooth and that circular particles (orange arrows in Fig. 1b) were attached to the surfaces of SF@PDA@LIP. ImageJ software analysis (Fig. 1d) indicated that the SF diameter was $837.15 \pm 201.21 \mathrm{~nm}$. After PDA modification, the fiber diameter of SF@PDA was reduced to $720.95 \pm 196.52 \mathrm{~nm}$, while the that of SF@PDA@LIP was $801.7 \pm 177.75 \mathrm{~nm}$ due to $\mathrm{NH}_{2}$-LIP attachment. Analysis of AFM images using NanoScope analysis software revealed that the fiber roughness (Ra) of SF and SF@PDA was $380 \mathrm{~nm}$ and $374 \mathrm{~nm}$, respectively. (Fig. 1c) The Ra greatly increased to $449 \mathrm{~nm}$ for SF@PDA@LIP after $\mathrm{NH}_{2}$-LIP attachment to SF@PDA. These changes confirm that $\mathrm{NH}_{2}$-LIPs were successfully grafted onto the surface of SF@PDA. $\mathrm{NH}_{2}$-LIPs labeled by coumarin, a fluorescent dye, were grafted onto the surface of SF and SF@PDA and observed using fluorescence microscopy (Fig. 1e). There were almost no bright green spots around SF (Fig. 1e iii) and many bright green spots around SF@PDA (Fig. 1e i), which remained around the latter after rinsing three times with deionized water (Fig. 1e ii), indicating that the $\mathrm{NH}_{2}$-LIPs were firmly immobilized onto the SF@PDA surface.

Figure 2a shows the chemical structural variation of elements on the surface of the fibrous membrane. The mechanical properties of SF, SF@PDA, and SF@PDA@LIP were measured to determine the effects of the PDA coating and $\mathrm{NH}_{2}$-LIP grafting on the mechanical properties of SF. Stress-strain curves (Fig. 2b) revealed tensile strengths of $1.95 \pm 0.24,2.58 \pm 0.27$, and $2.87 \pm 0.21 \mathrm{MPa}$ for SF, SF@PDA, and SF@PDA@LIP, respectively, indicating a dramatic improvement in tensile strength through PDA coating. The Young's modulus of SF, SF@PDA, and SF@PDA@LIP was 0.026, 0.0087, and $0.088 \mathrm{MPa}$, respectively (Fig. 2c). The PDA and $\mathrm{NH}_{2}$-LIPs on the SF fiber membrane surface increased the deformation resistance of SF. Figure $2 \mathrm{~d}$ shows the hydrophilicity of SF, SF@PDA, and SF@PDA@LIP. The WCA of the SF membrane was $64^{\circ}$ and was greatly reduced to near $0^{\circ}$ by PDA modification and LIP grafting. These 


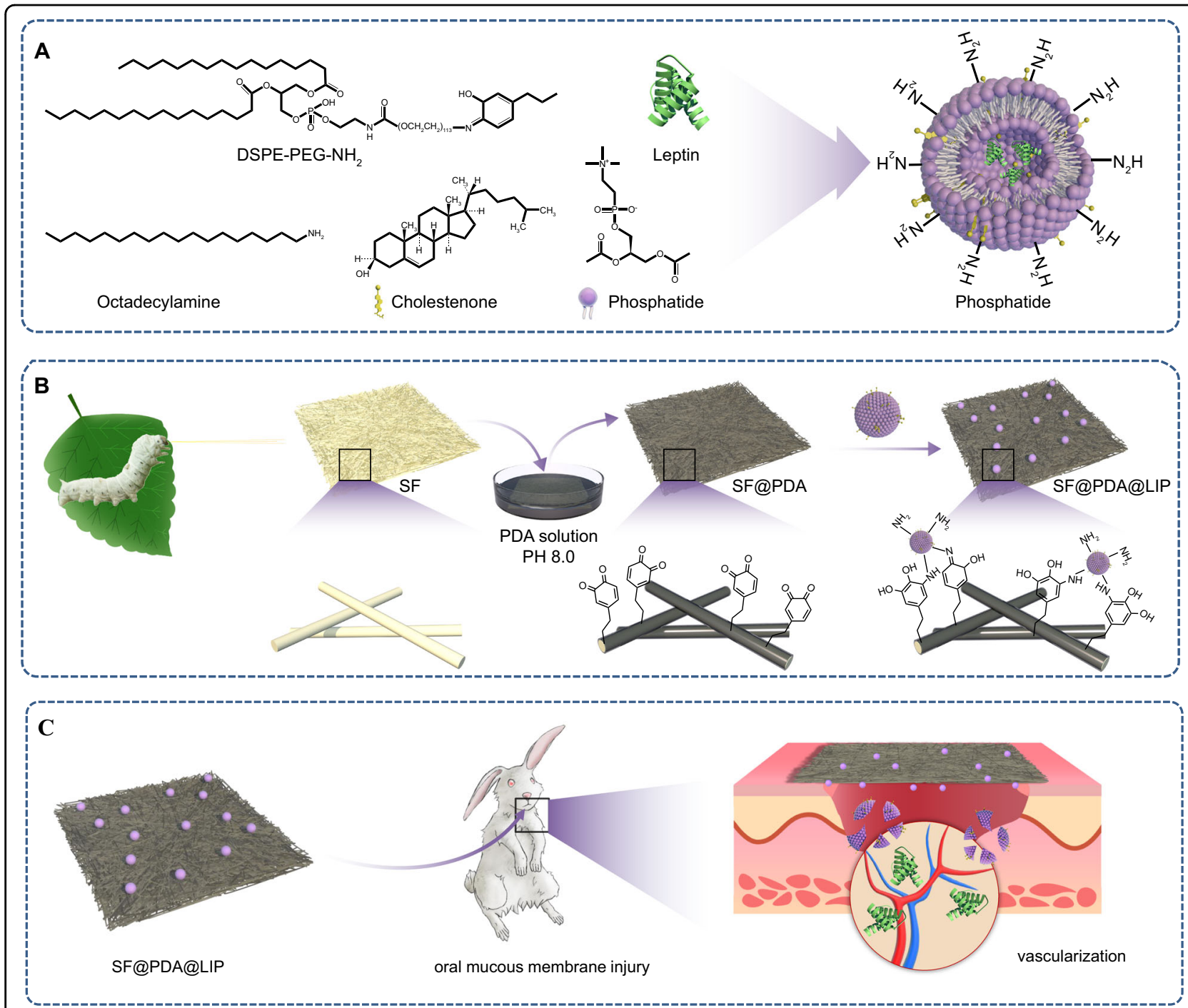

Scheme 1 a Schematic preparation of $\mathrm{NH}_{2}$-LIPs. b Illustration of the building process of the SF, SF@PDA, and SF@PDA@LIP membranes. c SF@PDA@LIP loaded with leptin and used to cover oral mucous membrane injury to promote angiogenesis.

changes confirmed that PDA successfully covered the SF surfaces and indicated that SF@PDA and SF@PDA@LIP are superhydrophilic.

XPS analysis of SF, SF@PDA, and SF@PDA@LIP revealed peaks at 286.0, 400.0, and $532.0 \mathrm{eV}$, indicating that all three fibers contain $\mathrm{C}, \mathrm{N}$, and $\mathrm{O}$, respectively. However, the areas below the N peaks for SF@PDA and SF@PDA@LIP were larger than that of SF because of the presence of PDA (Fig. 3b). The proportion of $\mathrm{C}=\mathrm{O}$ in the $C$ peak of SF was $14.43 \%$ and increased to $21.05 \%$ after PDA modification. The $\mathrm{C}=\mathrm{O}$ proportion on the surface of SF@PDA@LIP decreased to 0.13\%. The highest proportion of $\mathrm{C}$ in SF@PDA@LIP was C-C bonds, $86.5 \%$ (Fig. 3c, e g). The proportion of $\mathrm{C}=\mathrm{O}$ bonds in the $\mathrm{O}$ peak of SF was $60.66 \%$, and that of $\mathrm{C}-\mathrm{OH}$ bonds was $37.38 \%$. The proportions of $\mathrm{C}=\mathrm{O}$ and $\mathrm{C}-\mathrm{OH}$ in SF@PDA increased to $62.56 \%$ and $30.65 \%$, respectively, due to the presence of PDA. The proportions of $\mathrm{C}=\mathrm{O}$ and $\mathrm{C}-\mathrm{OH}$ in SF@PDA@LIP were $40.76 \%$ and $53.61 \%$, respectively (Fig. $3 \mathrm{~d}, \mathrm{f}, \mathrm{h}$ ). In addition,SF@PDA@LIP has a peak at $133.0 \mathrm{eV}$, indicating that SF@PDA@LIP surfaces contain P (Fig. 3b). The results show that $\mathrm{NH}_{2}$-LIPs were successfully grafted onto the surfaces of SF@PDA. Drug release is another important index for biomaterial evaluation. Figure 2e shows the release curves of $\mathrm{NH}_{2}$-LIPs on the surfaces of SF@PDA@LIP and LIPs, which present no significant difference. $(P>0.05)$ The leptin release rates of LIPs and $\mathrm{NH}_{2}$-LIPs at $48 \mathrm{~h}$ were 77.97 and $75.55 \%$, respectively, demonstrating that grafting $\mathrm{NH}_{2}$-LIPs to the surface of SF@PDA causes no significant 

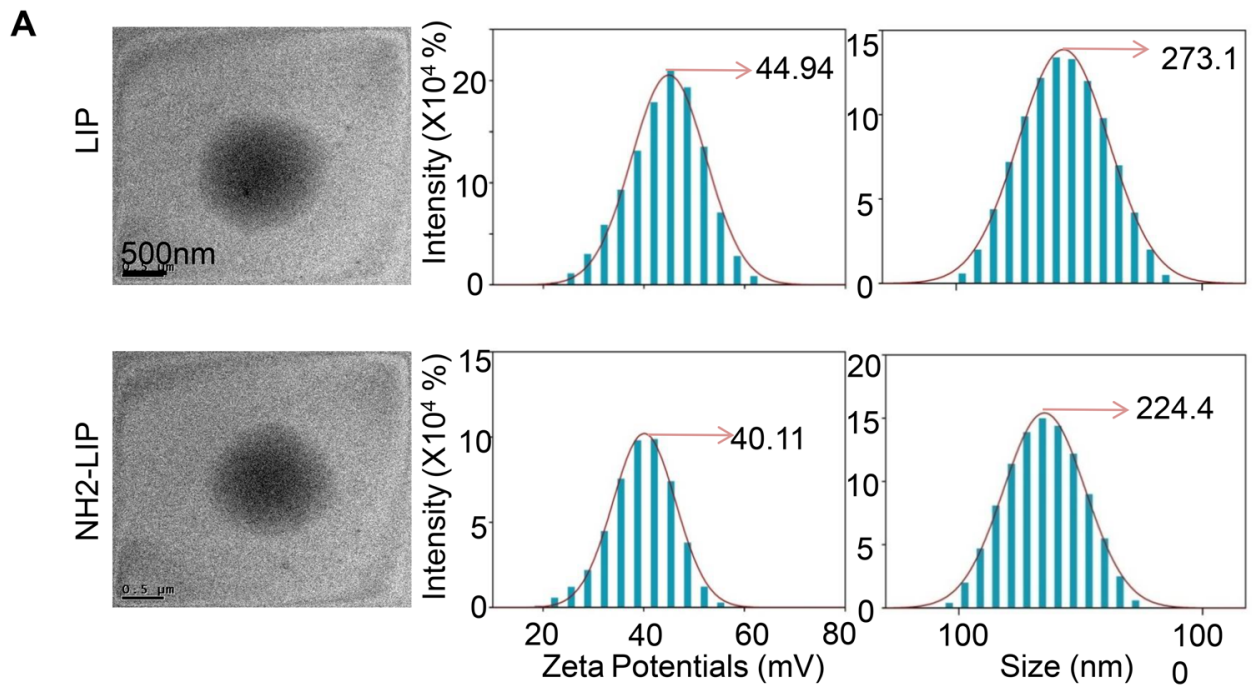

B
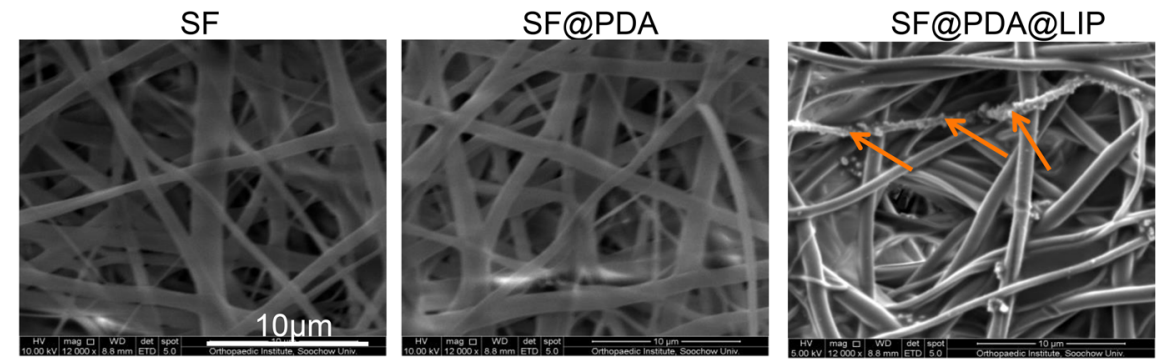

C
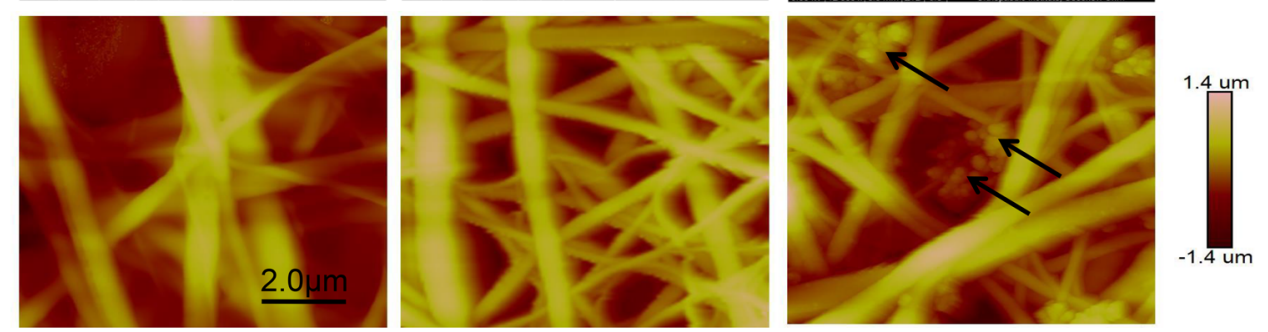

D
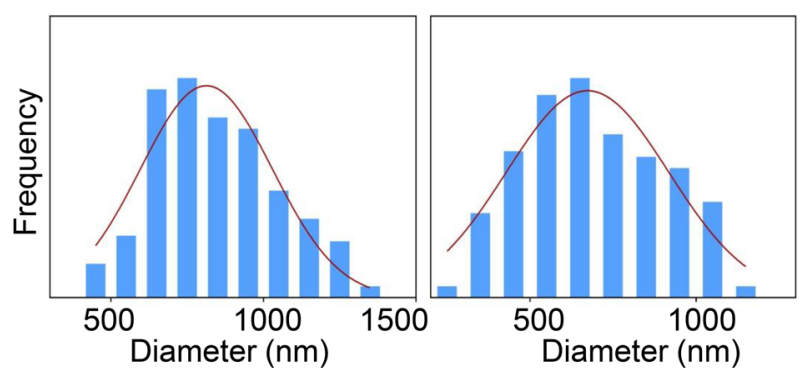

E
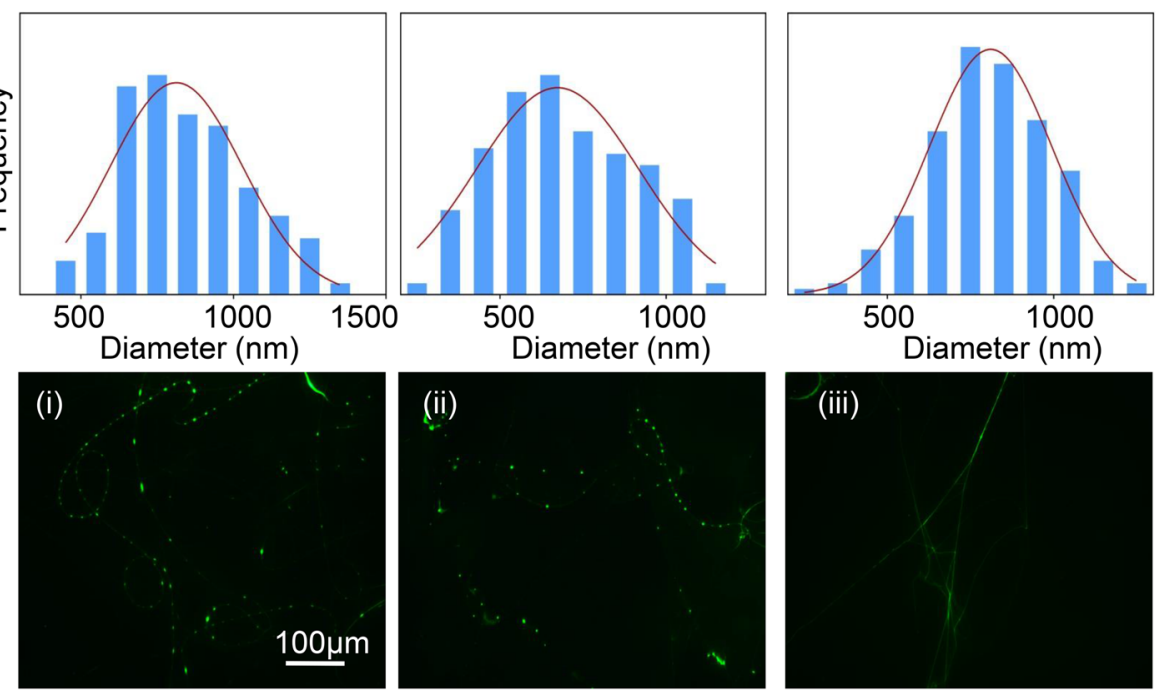

Fig. 1 Characterization of NH2-LIPs and different fibrous membranes. a TEM images, zeta potentials and size distributions of LIPS and NH2-LIPS. b SEM images, (c) AFM images and (d) diameter frequency distribution datagrams of SF, SF@PDA, and SF@PDA@LIP. e Coumarin-labeled NH2-LIPS grafted onto (i) SF@PDA fiber, then (ii) rinsed with deionized water three times, iii) SF fiber. 

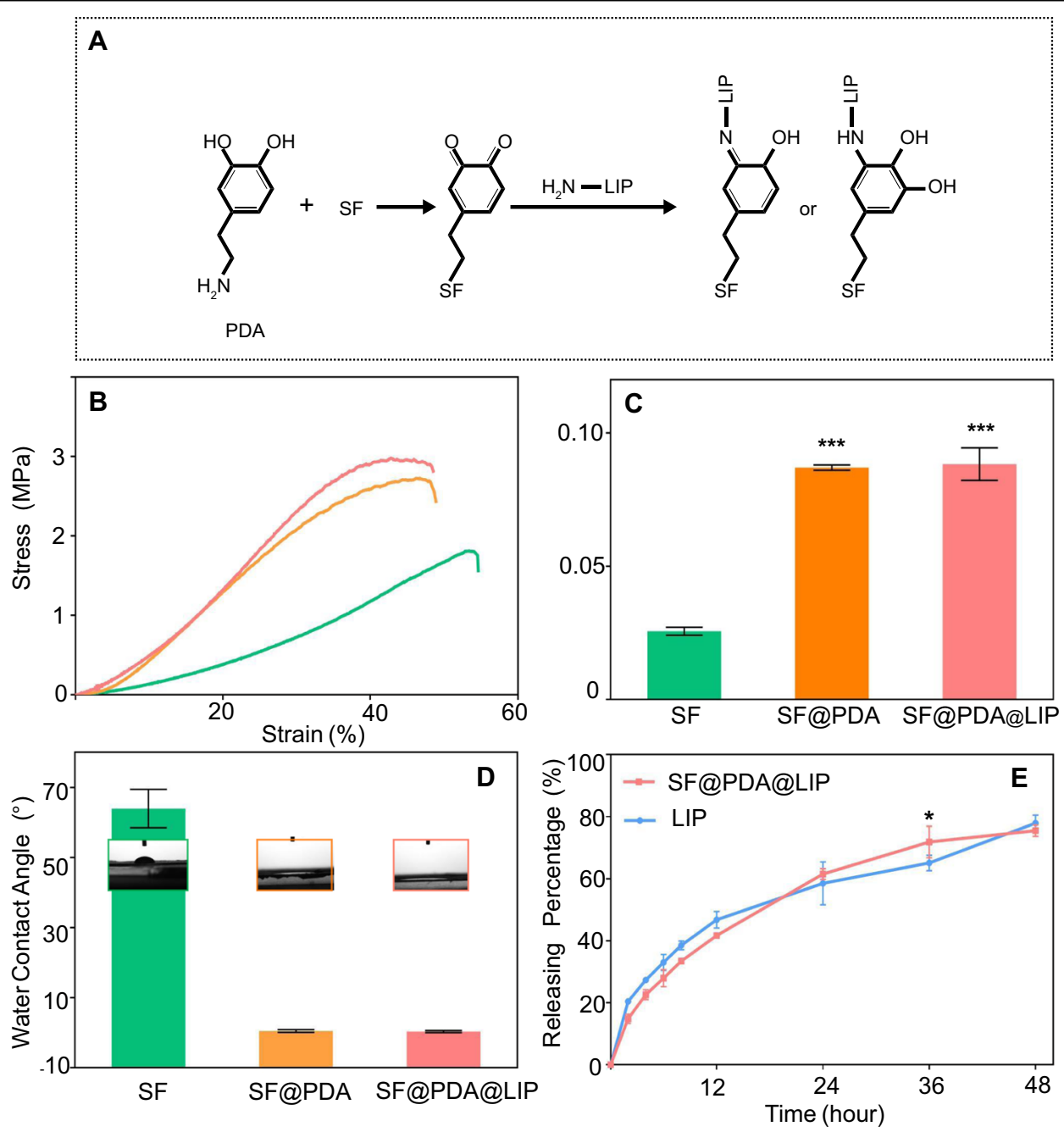

Fig. 2 Physicochemical properties of fibrous membranes. a Illustration of the chemical structure variation. b Stress-strain curves, (c) Young's modulus and (d) WCA of SF, SF@PDA, SF@PDA@LIP. e Release curves of leptin in LIPs and SF@PDA@LIP. (**p $<0.0005$ compared with SF).

differences in leptin release behavior compared to that of LIPs.

Rat fibroblasts were cultured on the fibrous membranes to evaluate the effects of the membranes on cell proliferation and adhesion. All fibrous membranes exhibited good biocompatibility after 3 days of culture, as cells on SF, SF@PDA, and SF@PDA@LIP appeared normal. As shown in Fig. 4a, the cytoskeletal spreading area of cells on SF was smaller than that of cells on SF@PDA and SF@PDA@LIP, with little difference in the $\alpha$-SMA levels between SF@PDA and SF@PDA@LIP. The average fluorescence intensities of $\alpha$-SMA as calculated by ImageJ were $0.017,0.066$, and 0.067 for SF, SF@PDA, and SF@PDA@LIP, respectively (Fig. 4d). The average fluorescence intensity of integrin expressed by cells cultured with fiber membranes containing PDA (SF@PDA and SF@PDA@LIP) was 0.63 and 0.65 , respectively, while on $\mathrm{SF}$, only the average fluorescence intensity was 0.023 .
Such differences are likely because PDA facilitates cell adhesion (Fig. 4b and e). Figure 4c depicts the proliferation rates of cells on each fiber type and reveals that the growth rates of cells on SF@PDA@LIP and SF@PDA were higher than those of cells on SF. These results thus demonstrated that fibers containing PDA increase the growth and adhesion of fibroblasts.

To probe the angiogenic abilities of our test fibers, human umbilical vein endothelial cells (HUVECs) were cultured with leaching solutions of SF, SF@PDA, and SF@PDA@LIP. Figure 5a shows that after 5 h, HUVECs cultured in SF@PDA@LIP fiber leaching solution formed primary capillary-like networks, while HUVECs cultured in SF and SF@PDA solutions had inconspicuous tubular structures. A total of 38.67 and 35 mature junctions were formed on SF and SF@PDA, respectively, which is far lower than the 112.7 junctions formed on SF@PDA@LIP. After $10 \mathrm{~h}$, although the amount of tubular structures of 

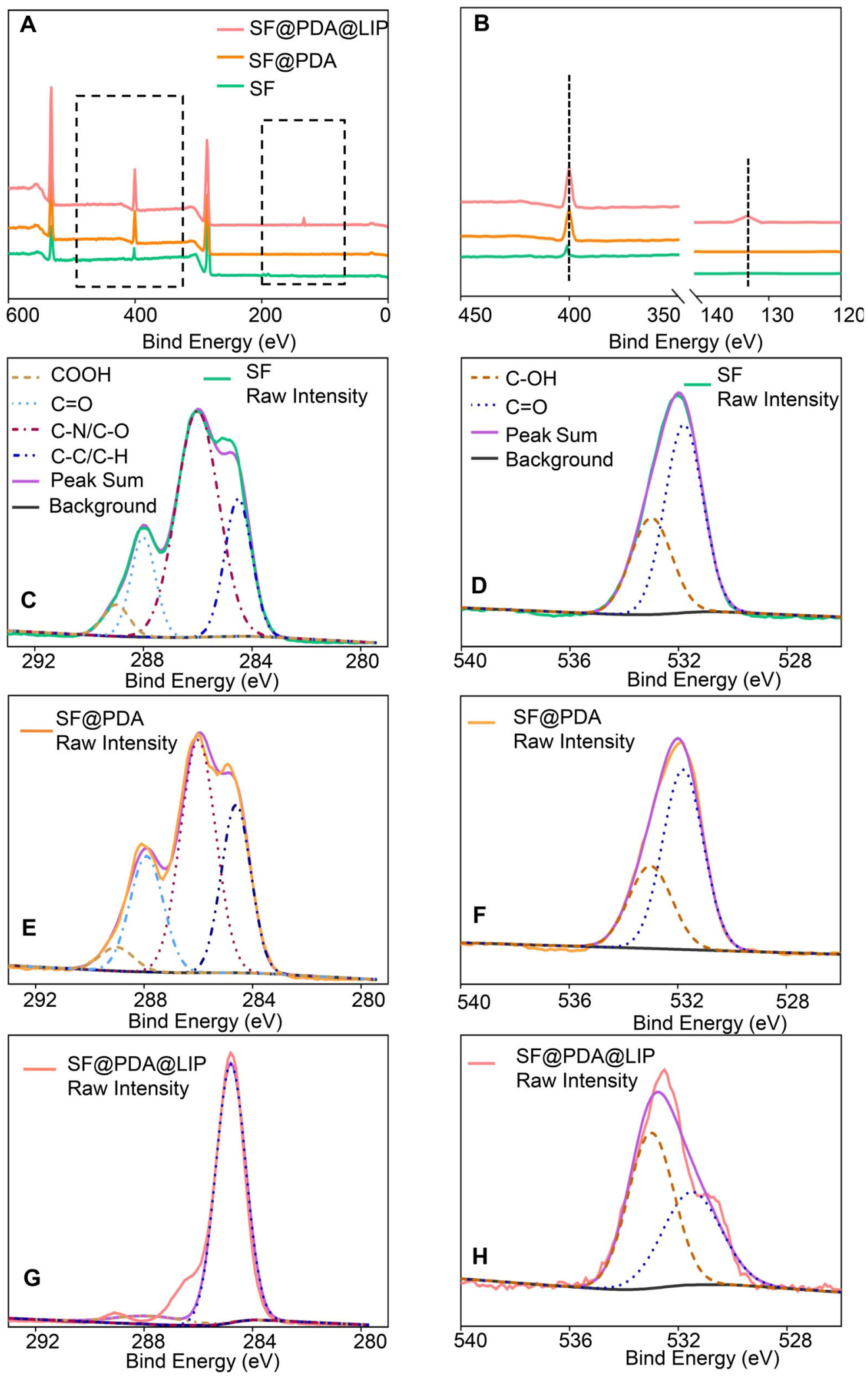

Fig. 3 XPS analyses of fibrous membranes. a XPS spectra and (b) detailed peaks at 400 eV and 133 eV of SF, SF@PDA, and SF@PDA@LIP. The fitting curves of C on the surface of (c) SF, (e) SF@PDA and (g) SF@PDA@LIP. The fitting curves of O on (d) SF, (f) SF@PDA, and (h) SF@PDA@LIP. 

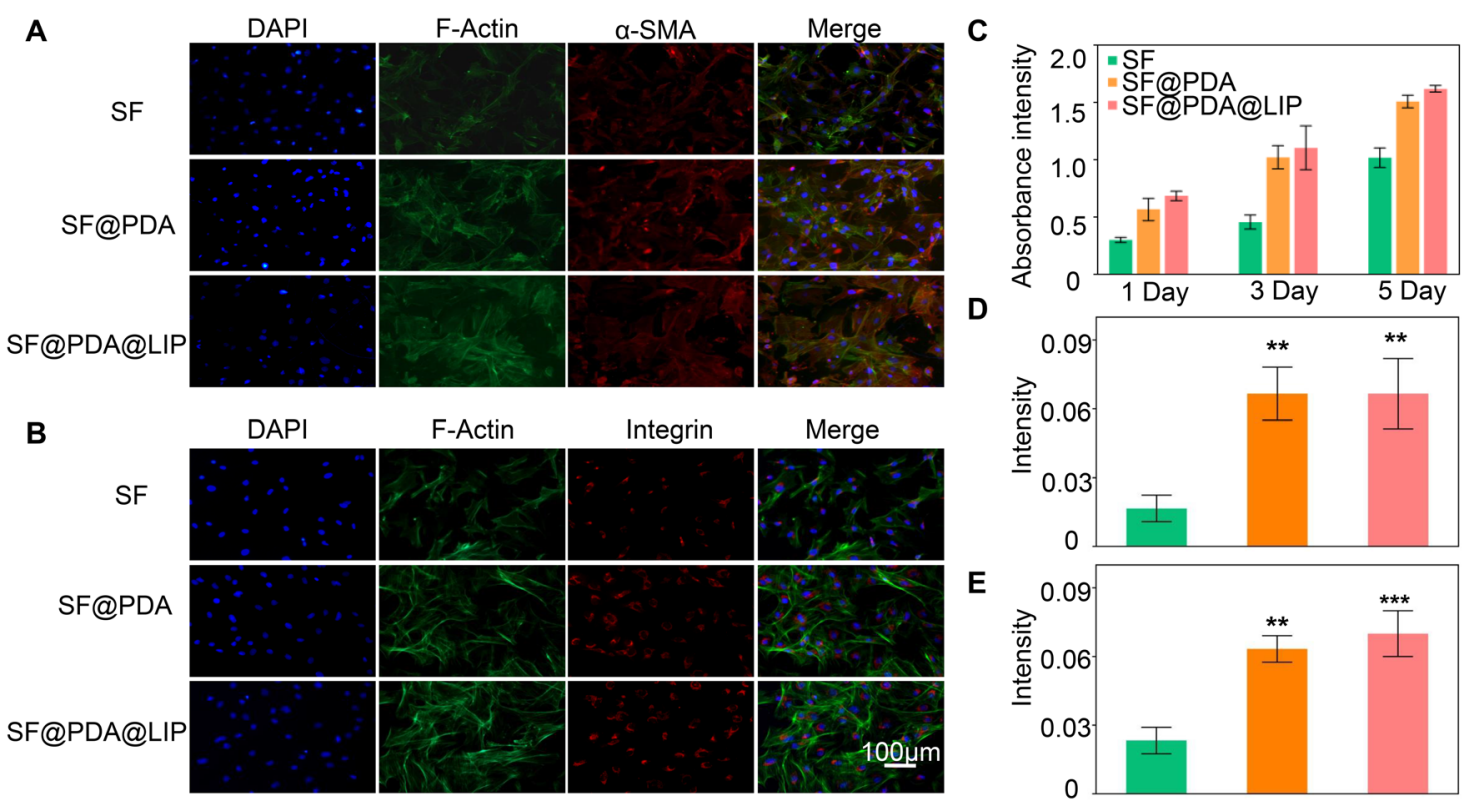

Fig. 4 Cell adhesion and proliferation assays of fibrous membranes. The expression of (a) a-SMA (red) and (b) integrin (red) in fibroblasts after coculture on SF, SF@PDA, and SF@PDA@LIP for 3 days. c Proliferation of fibroblasts cultured on fibrous membranes after 1, 3, and 5 days. The mean fluorescence intensity of (d) a-SMA and (e) vinculin in fibroblasts. $\left({ }^{* *} p<0.005,{ }^{* *} p<0.0005\right.$ compared with SF).

cells increased in all three groups, the number of mature junctions formed by cells on SF@PDA@LIP was still higher than that of cells in the other two groups (Fig. 5b). In addition, the number of meshes formed by cells in the SF@PDA@LIP leachate is higher than that in the SF and SF@PDA leachate (Fig. 5d). Similarly, in terms of mesh area (Fig. 5c) and tube length (Fig. 5e), cells on SF@PDA@LIP surpassed those on SF@PDA and SF at two time points. These results thus demonstrate that SF@PDA@LIP leachate, which contains leptin, facilitates the arrangement of HUVECs into tubular structures.

SF, SF@PDA, and SF@PDA@LIP were implanted into oral mucosal defect models in rabbits in order to evaluate the effects of fibrous membranes on oral defect repair. Figure 6a shows the conditions of damaged oral mucosa during recovery in rabbits treated with gauze (as a control), SF, SF@PDA, or SF@PDA@LIP at 3, 5, 7, and 14 days postoperation. The mucous membranes of all the rabbits largely recovered after 14 days; however, 3 days after the operation, wounds treated with fibrous membranes were more healed than wounds treated with gauze. In addition, the wounds covered with SF@PDA@LIP recovered the most, with the mucosal injury almost healed 7 days postsurgery, while wounds covered with SF or SF@PDA were less healed. At 3 days postoperation, the healing rates of wounds treated with SF@PDA and SF@PDA@LIP (Fig. 6b), as measured by ImageJ software, were $52.80 \%$ and $61.13 \%$, respectively, whereas SF-treated wounds showed $43.13 \%$ healing. In contrast, the healing rate of wounds treated only with gauze was 9.48, 22.06, and $47.72 \%$ at 3, 5, and 7 days postoperation, respectively. Although the healing rate of this control group reached 97.32\% 14 days postoperation, compared to fibrous membranes, gauze is not capable of promoting oral mucosa regeneration. The healing rates of mucosal injuries treated with SF@PDA@LIP were 81.09, 87.53, and $99.71 \%$ at 5,7 , and 14 days postoperation, respectively, representing the best promotion of injury healing among the fibrous membranes tested. These results show that SF membranes can promote wound repair and that the combination of PDA and $\mathrm{NH}_{2}$-LIPs can improve the defect repair abilities of SF membranes.

Mucosa microstructures were stained with H\&E (Fig. 7a). The control group mucosa had higher levels of inflammatory cell infiltration 5 days postoperation, and the epithelial structure at 14 days postoperation was unclear. The SF-treated mucosa formed unclear epithelial stratification with poorer cell morphology and higher inflammatory cell infiltration 14 days postoperation than those of SF@PDA-treated and SF@PDA@LIP-treated mucosa. At 3 days postoperation, newly formed mucosa produced during treatment with SF@PDA@LIP showed distinct epithelial stratification, the basal layer thickness was uniform, epithelial spikes were obvious, and cells appeared healthy. Collagen fibers formed under the epithelia repaired by SF@PDA@LIP and SF@PDA appeared orderly based on Masson staining (Fig. 7b). For the control group, recovered submucosal collagen fibers were proliferated and disordered, and there were few new small blood vessels. In contrast, SF@PDA@LIP-treated injuries 


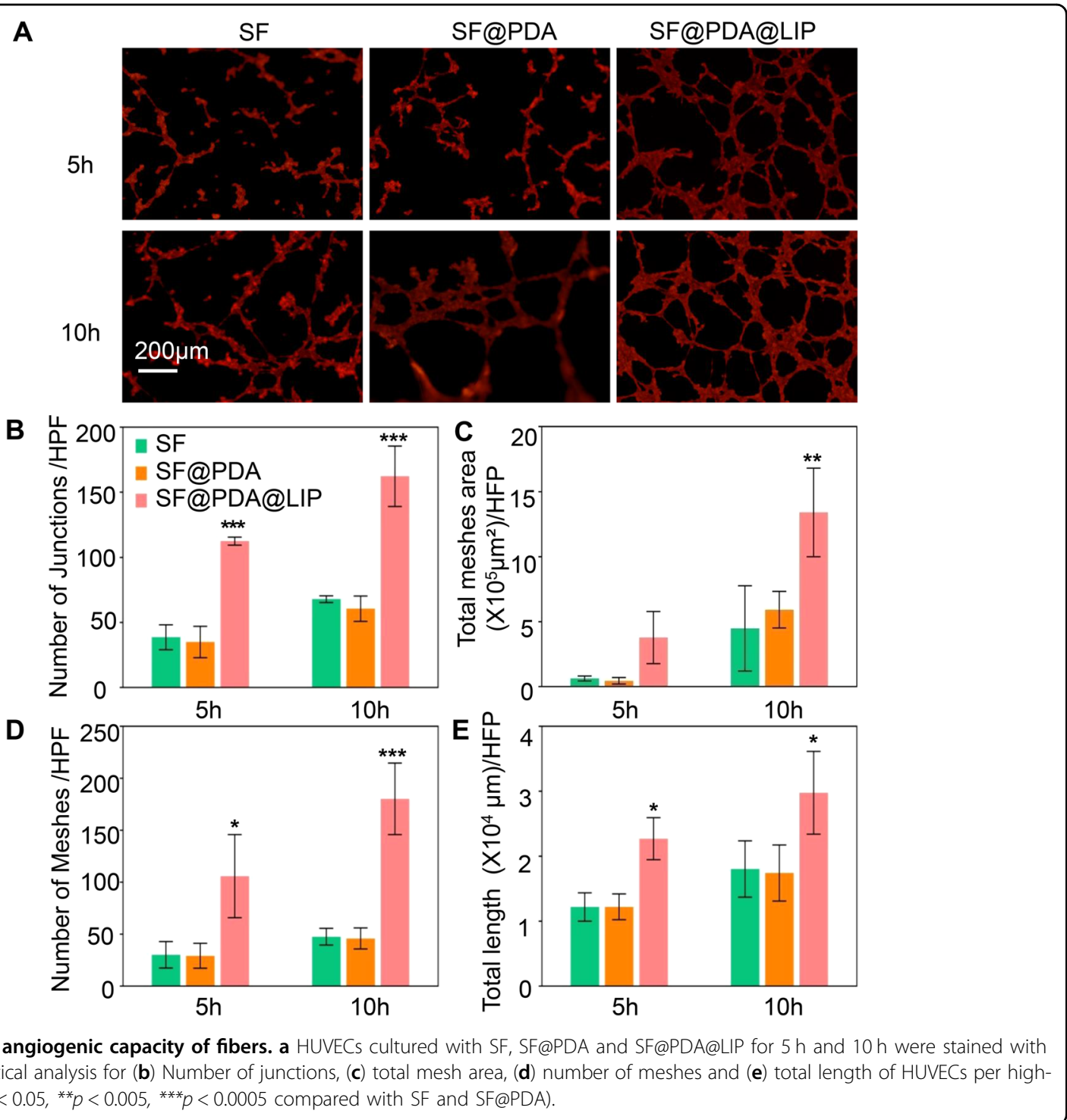

displayed new small blood vessels in the submucosa. The thickness of the new mucous membrane treated with the fibrous membrane, especially SF@PDA@LIP, reached $114.5 \mu \mathrm{m}$ and was thicker than that in the control group 5 days after the operation. Although the thickness in all groups increased at 14 days after operation, new mucosa repaired by SF@PDA@LIP was still the thickest (Fig. 7c). In addition, on the 14th day after operation, the number of epithelial spike processes in the fibrous membrane group was greater than that in the control group. The results were consistent with the thickness of the upper mucous membrane (Fig. 7d). In Fig. 8a, the brownish yellow coloring indicates the presence of CD34 in newly produced mucosa, representing newly generated blood vessels (black arrows). Visually, SF@PDA@LIP-treated mucosa showed a distinct CD34 signal, and the signal strength was higher than that in the mucosa of the control, SF, and SF@PDA groups (Fig. 8b). Twenty-nine blood vessels per high-power visual field were found in the mucous membrane treated with SF@PDA@LIP, while only 9.67, 9.33, and 10.67 were found in the control, SF, and SF@PDA groups, respectively. Obviously, the CD34 signal in the SF@PDA@LIP group was stronger than that in the other three groups. (Fig. $8 \mathrm{c}$ ) The average optical density (AOD) of CD34 in the mucosa was calculated. The AOD of the CD34 signal of the mucous membrane treated with the SF@PDA@LIP fiber membrane was 0.69 , which was the highest among the four groups (Fig. 8d).

It is well known that the destruction of blood vessels after severe injury to large areas of the oral mucosa destroys the blood supply in the center of the wound. At the same time, hypoxia and the infiltration of a large number of inflammatory factors stimulate the growth of 


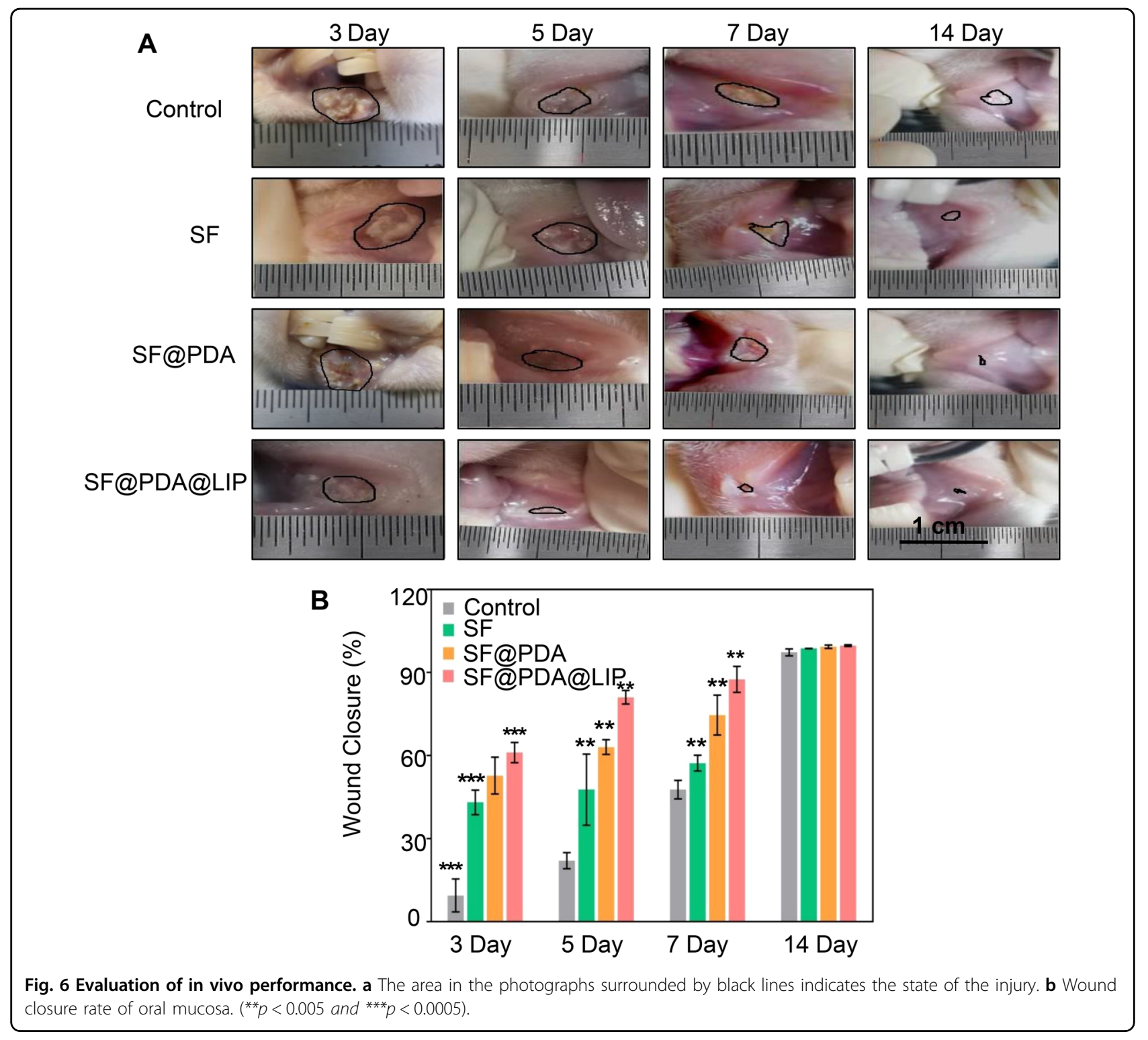

blood vessels at the edge of the wound towards the center. However, due to the severity and large size of these types of injury, the surrounding blood vessels cannot expand to completely cover the wound, the damaged mucous membranes have poor blood circulation, and the nutrient supply is insufficient; therefore, wound healing is slow. Neovascularization is thus an important requirement for complete tissue healing ${ }^{49}$. Due to developments in tissue engineering, increasing numbers of biological scaffolds are available for transplantation into a wide range of complex damaged tissues to facilitate tissue repair. Unfortunately, these current biological scaffolds, even if capable of directing cell growth, are limited in their utility due to an inability to promote angiogenesis. Biological scaffolds that promote angiogenesis have obvious advantages for increasing tissue healing rates.
In this study, to stimulate vascular regeneration and accelerate the recovery of damaged oral mucosa, we used electrospinning to produce fiber membranes capable of promoting angiogenesis. Electrospinning is a popular method for preparing fiber membranes, as it is able to maintain the fiber membrane porosity and produce fiber membrane thicknesses of less than $150 \mu \mathrm{m}$, thereby ensuring the transport of sufficient oxygen and nutrition in wounds ${ }^{50}$. Fibrous membranes manufactured by electrospinning are thus suitable for oral cavities that have inherently limited space. The three-dimensional structure of electrospun fiber membranes is similar to that of the ECM, thus favoring cell proliferation and adhesion ${ }^{50,51}$. In addition, silk fibroin is an excellent material for preparing these fibrous membranes because it is a natural biomaterial with good biocompatibility and anti-inflammatory 


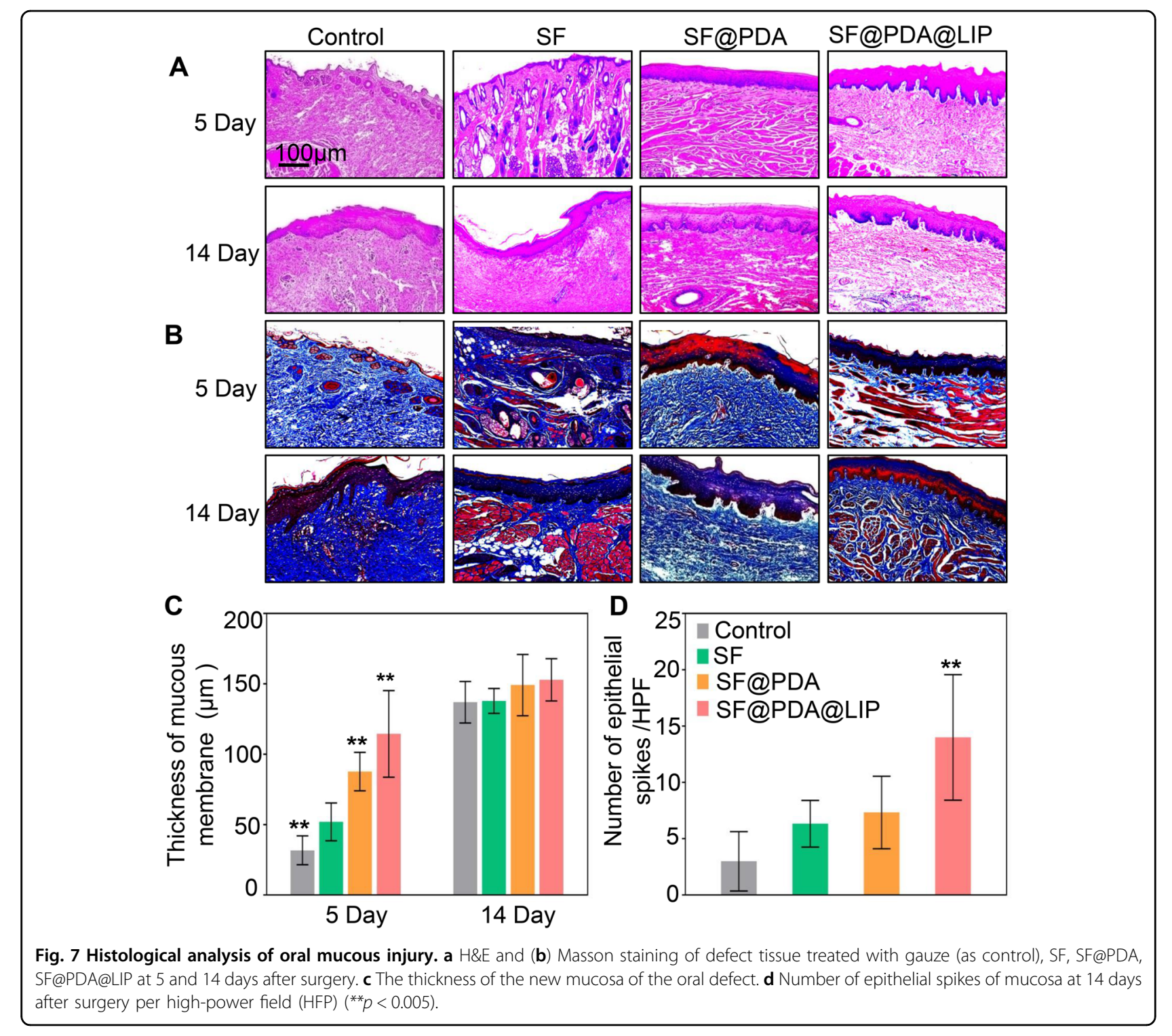

properties and is able to promote the growth and proliferation of fibroblasts and epithelial cells. We utilized PDA as a link for grafting $\mathrm{NH}_{2}$-LIPs onto the surface of SF membranes to create electrospun fibrous membranes that are able to locally release leptin to stimulate blood vessel regeneration in damaged mucous membranes, thus producing SF membranes capable of promoting angiogenesis.

PDA is a nontoxic protein based on the compound DOPA used by mussels that can adhere to moist reefs and is thus deposited on multiple types of surfaces ${ }^{46}$. Under alkaline conditions, catechol groups of PDA can be oxidized to quinone groups, which can react with amino groups on the surface of $\mathrm{NH}_{2}$-LIPs by a Michael addition reaction or Schiff base reaction ${ }^{13}$. At present, there is no clear evidence that PDA has a great effect on the microstructure of fibrous membranes ${ }^{13,35,46}$. The diameter of
SF@PDA was slightly smaller than that of SF. We speculated that the reason may be related to the influence of randomly selected fibers. The difference in the diameters of SF and SF@PDA was not statistically significant $(P=0.3441)$ (Fig. 1b). Some studies have shown that the surface of nanoparticles is smoother after being coated with $\mathrm{PDA}^{46,52}$. It was possible that the roughness of SF@PDA was reduced because PDA filled the depression of SF. Because of the quinonegroups generated from catechol groups after the oxidation reaction in an alkaline environment, the proportion of $\mathrm{C}=\mathrm{O}$ bonds in SF@PDA was increased, indicating that PDA successfully modified SF. A higher N peak value was observed for SF@PDA@LIP than for the other fibers tested, confirming that PDA successfully modified the surface of SF. The reason for the increased proportion of $\mathrm{C}-\mathrm{C}$ and $\mathrm{C}-\mathrm{OH}$ bonds in 


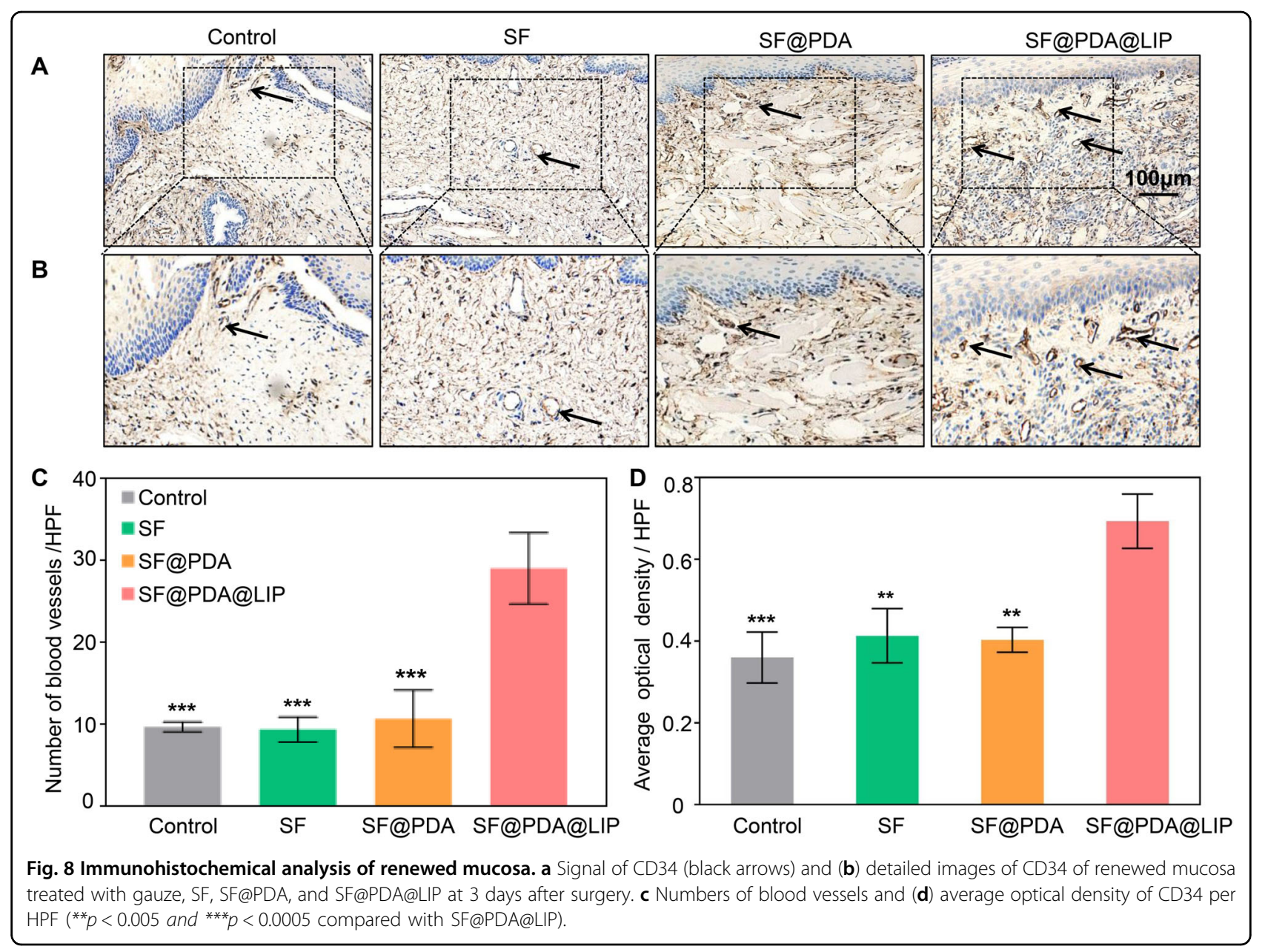

SF@PDA@LIP is the number of C-C structures in DSPEPEG- $\mathrm{NH}_{2}$ and the reaction between the amino groups of LIPs and $\mathrm{C}=\mathrm{O}$ bonds of PDA, respectively. In addition, the presence of the P peak of SF@PDA@LIP indicated that LIP was successfully grafted to the fiber surface (Fig. 3). The immobilization of nanoparticles on SF@PDA@LIP (Fig. 1a) and the increased roughness demonstrated that $\mathrm{NH}_{2}$-LIPs were successfully grafted onto the surface of SF via PDA. Fluorescence microscopy also confirmed that the coumarin-labeled $\mathrm{NH}_{2}$-LIPs accumulated around SF@PDA fibers (Fig. 1e). The deposition of PDA on SF glued the fibers together so that the fiber stability and deformation resistance were increased. $\mathrm{NH}_{2}$-LIPs also strengthen the fibrous mechanical properties by connecting to $\mathrm{PDA}^{53,54}$. The WCA of SF@PDA and SF@PDA@LIP decreased due to the PDA coating, which contains multiple functional groups and is strongly hydrophilic (Fig. 2d). Saliva secretions, eating, and other factors create a humid environment within the oral cavity, potentially affecting the superhydrophilic SF@PDA@LIP fiber membrane, which must be addressed in future work. When a mucous membrane injury enters the proliferation phase, stimulating endothelial cell proliferation to promote angiogenesis as soon as possible provides nutrition for epithelial regeneration. $\mathrm{NH}_{2}$-LIPs prevent the destruction of leptin's biological activity by both organic solvents and oral cavity enzymes and quickly release leptin into wounded tissues, thereby accelerating angiogenesis in the early stages of healing. $\mathrm{NH}_{2}$-LIPs can also be successfully immobilized onto the surfaces of SF@PDA through reactions between $\mathrm{NH}_{2}$-LIPs and PDA that have been deposited on SF surfaces. This immobilization mechanism has little effect on the overall structure of LIPs and thus does not affect the cleavage of LIPs or the leptin release rate (Fig. 2e).

The surface chemical structure of the fiber plays a key role in affecting cell behavior and response. The changes in hydrophilicity on the SF surface can affect protein adsorption and thus cell adhesion ${ }^{46}$. According to previous studies, PDA does not hinder the proliferation of fibroblasts, osteoblasts, neurons or endothelial cells. In addition, many studies have shown that compared with the original matrix, the PDA-coated matrix can promote cellular adhesion and proliferation in a substance- 
independent manner ${ }^{55}$. The cell experiments revealed that the number of cells cultured on SF@PDA and SF@PDA@LIP was greater than that on SF, which also proved that PDA has the function to promote cell proliferation. PDA has great potential for cell adhesion. Yang et al. coated PDA on the surface of yeast cells and fixed the cells on a selected matrix via covalent attachment. As a result, PDA could control cell division and protect cells from external attack ${ }^{55}$. PDA can simply connect to amino or thiol groups on the cell membrane and can easily attach cells to the surface of the fibrous membrane. Cellular staining showed that the surface area of fibroblasts on SF@PDA and SF@PDA@LIP was larger than that on SF. The presence of PDA on the fibrous membrane was favorable for cell adhesion according to the fluorescence intensity results of fibroblasts. (Fig. 4)

The encapsulation of leptin by $\mathrm{NH}_{2}$-LIPs is an important component of SF@PDA@LIPs and promotes vascularization in wounds. SF@PDA consists of natural silk fibroin and nontoxic PDA and has good cytocompatibility. These fibrous membranes have no obvious detrimental effects on cell proliferation ${ }^{35,46}$. Leptin is transcribed from the $\mathrm{OB}$ gene on chromosome 7 and functions as an angiogenesis-related factor. Leptin has been shown to be involved in brain development, synaptic transmission, and angiogenesis after cerebral ischemia-reperfusion injuries and is an important factor in the treatment of such injuries $^{25}$. In the last century, Boulumie et al. discovered that endothelial cells express leptin receptors that were stimulated by leptin to induce cells to form tube-like structures $^{56}$. In experiments with HUVEC tube formation, tubular structures of endothelial cells cultured with SF@PDA@LIP were more complete than in cells cultured with either SF@PDA or SF (Fig. 5). Such effects are due to leptin release from $\mathrm{NH}_{2}$-LIPs, which increases the expression of angiogenic factors and promotes the arrangement of HUVECs into tubular structures.

The mucosa is the internal tissue of the oral cavity and is located at the beginning of the digestive tract. If serious oral mucosa injuries cannot be dealt with quickly, they can heal poorly, form scar tissue, and affect oral function. Silk fibroin and PDA have been widely used in tissue engineering because of their good biocompatibility ${ }^{57,58}$. Gil et al. applied silk fibroin as a wound dressing to skin wounds and found that compared with the blank group, the silk fibroin dressing provided sufficient space for cell migration at the wound site and promoted the growth of epithelial cells ${ }^{59}$. In the rabbit oral mucosal defect model, because SF promotes epithelial cell growth, has good biocompatibility, and is anti-inflammatory, wounds treated with SF healed better than those treated with gauze and showed clear stratification of the mucosal structure. In addition, there was less infiltration of inflammatory cells in the submucosa with SF treatment than with gauze treatment (Fig. 7). Oral mucosa is typically in close contact with air and food, making it easy for bacteria to survive and multiply inside the oral cavity and increasing the risk of infection of damaged mucosa. However, PDA is an antibacterial agent and effectively prevents wound infections while accelerating mucosal regeneration. Mao and his colleagues deposited PDA on cotton fabrics to produce an antibacterial dressing. After the antibacterial activity test, Escherichia coli (E. coli) was completely killed by PDA-modified cotton fabrics. More interestingly, the dressing was still able to eliminate $99 \%$ of E. coli after 30 washes. PDA has great prospects in making antibacterial bandages to prevent wound infections ${ }^{46}$. In addition, the hydrophilicity of PDA enhances adhesion between fibers and cells; thus, SF@PDA and SF@PDA@LIP can repair mucosa better than SF. Leptin in SF@PDA@LIP released from the ruptured $\mathrm{NH}_{2}$-LIPs activates multiple pathways, including the STAT3 and MAPK pathways, to stimulate the high expression of angiogenic factors and increase blood vessel formation in the wound. Histological analysis showed the presence of many new small blood vessels, and the strongest CD34 signal was observed in injured mucosa treated with SF@PDA@LIP (Fig. 8). The healing rate of wounds treated with SF@PDA@LIP was the fastest, with $>50 \%$ wound healing 3 days after surgery. According to our results, we propose that SF@PDA@LIP containing PDA links for grafting $\mathrm{NH}_{2}$-LIPs onto SF membranes can promote blood vessel formation in the center of wounds and accelerate oral mucosa regeneration. However, more studies are needed to determine the optimal dose of leptin loaded in fibrous membranes and the mechanisms by which leptin promotes angiogenesis.

In addition, our study has few limitations. First, it is hard to accurately control the dosage of leptin loaded because it cannot be ensured that all $\mathrm{NH}_{2}$-LIPs can bond onto PDA. Second, the release direction of leptin cannot be controlled, and it is impossible to ensure that all leptin goes into the defect. Third, the fibrous membranes need suitable pressure to be fixed in the mucosal defects, and the oral space of rabbits is small, so there is a limitation in the in vivo experiments.

\section{Conclusions}

$\mathrm{NH}_{2}$-LIPs grafted onto the surfaces of electrospinningproduced SF membranes via conjugation with PDA can locally release leptin to stimulate blood vessel regeneration and promote healing of damaged oral mucosa. PDA modification and $\mathrm{NH}_{2}$-LIP grafting had no significant effect on the morphology of SF but did improve the stability and mechanical properties of SF. In addition, the release behavior of $\mathrm{NH}_{2}$-LIPs attached to the surface of SF was not affected by reactions between $\mathrm{NH}_{2}$-LIPs and PDA. Oral mucosa tissue sections from rabbits treated 
with SF membranes loaded with leptin showed clear stratification of new mucosa, normal shaped epithelial spikes, uniform basal layers, and an obvious CD34 signal. In summary, we used PDA to graft $\mathrm{NH}_{2}$-LIPs onto the surface of SF membranes in order to promote the vascularization of SF membrane surfaces, which is expected to meet the current needs of clinical oral mucosal injury repair.

\section{Acknowledgements}

This research was funded by the National Natural Science Foundation of China (81671028 and 51873107), Shanghai Municipal Health and Family Planning Commission (201840027), Shanghai Municipal Education CommissionGaofeng Clinical Medicine Grant Support (20171906) and Shanghai Jiao Tong University "Medical and Research" Program (ZH2018ZDA04). The funding agencies did not have a role in the study design, study execution or data interpretation.

\section{Author details}

'Department of Stomatology, The First Affiliated Hospital of Soochow University, 899 Pinghai Road, 215006 Suzhou, Jiangsu, China. ${ }^{2}$ Department of Orthopaedic Surgery, The First Affiliated Hospital of Soochow University, 899 Pinghai Road, 215006 Suzhou, Jiangsu, China. ${ }^{3}$ Department of Orthopedics, The First Affiliated Hospital of Soochow University, Orthopedic Institute, Soochow University, 708 Renmin Road, 215007 Suzhou, Jiangsu, China. ${ }^{4}$ Department of Maxillofacial Surgery, The Affiliated Hospital of Yangzhou University, Yangzhou University, 368 Hanjiang Middle Road, 225000 Yangzhou, Jiangsu, China. ${ }^{5}$ Shanghai Institute of Traumatology and Orthopaedics, Shanghai Key Laboratory for Prevention and Treatment of Bone and Joint Diseases, Ruijin Hospital, Shanghai Jiao Tong University School of Medicine, 197 Ruijin Second Road, 200025 Shanghai, China

\section{Conflict of interest}

The authors declare that they have no conflict of interest.

\section{Publisher's note}

Springer Nature remains neutral with regard to jurisdictional claims in published maps and institutional affiliations.

Received: 30 October 2019 Revised: 19 February 2020 Accepted: 14 March 2020.

Published online: 5 June 2020

\section{References}

1. Zhang, J. \& Hughes, S. Role of the ephrin and Eph receptor tyrosine kinase families in angiogenesis and development of the cardiovascular system. J. Pathol. 208, 453-461 (2006).

2. Yao, D. et al. Regulating coupling efficiency of REDV by controlling silk fibroin structure for vascularization. ACS Biomater. Sci. Eng. 3, 3515-3524 (2017).

3. $\mathrm{Fu}, \mathrm{J}$. \& Wang, D. A. In situ organ-specific vascularization in tissue engineering. Trends Biotechnol. 36, 834-849 (2018).

4. Tian, T., Zhang, T., Lin, Y. \& Cai, X. Vascularization in craniofacial bone tissue engineering. J. Dent. Res. 97, 969-976 (2018).

5. Qazi, T. H., Mooney, D. J., Pumberger, M., Geissler, S. \& Duda, G. N. Biomaterials based strategies for skeletal muscle tissue engineering: existing technologies and future trends. Biomaterials 53, 502-521 (2015).

6. Caillaud, M., Richard, L., Vallat, J. M., Desmouliere, A. \& Billet, F. Peripheral nerve regeneration and intraneural revascularization. Neural Regen. Res. 14, 24-33 (2019).

7. Kreimendahl, F. et al. Macrophages significantly enhance wound healing in a vascularized skin model. J. Biomed. Mater. Res. A 107, 1340-1350 (2019).

8. Sun, X., Altalhi, W. \& Nunes, S. S. Vascularization strategies of engineered tissues and their application in cardiac regeneration. Adv. Drug Deliv. Rev. 96, 183-194 (2016).
9. Vatsyayan, A., Adhyapok, A. K., Debnath, S. C. \& Malik, K. Reconstruction and rehabilitation of short-range gunshot injury to lower part of face: A systematic approach of three cases. Chin. J. Traumatol. 19, 239-243 (2016).

10. Kim, T. G., Shin, H. \& Lim, D. W. Biomimetic scaffolds for tissue engineering. Adv Funct. Mater. 22, 2446-2468 (2012).

11. Chen, C. et al. Bioinspired hydrogel electrospun fibers for spinal cord regeneration. Adv. Funct. Mater. 29, 1806899 (2019).

12. Podlipec, R. et al. Molecular mobility of scaffolds' biopolymers influences cell growth. ACS Appl. Mater. Inter 6, 15980-15990 (2014).

13. $\mathrm{Xu}, \mathrm{Y}$. et al. Hierarchical micro/nanofibrous bioscaffolds for structural tissue regeneration. Adv. Healthc. Mater. 6, 1601457 (2017).

14. Yin, S., Zhang, W., Zhang, Z. \& Jiang, X. Recent advances in scaffold design and material for vascularized tissue-engineered bone regeneration. Adv. Healthc. Mater. 8, 1801433 (2019).

15. Qasim, S. B. et al. Electrospinning of chitosan-based solutions for tissue engineering and regenerative medicine. Int. J. Mol. Sci. 19, 1-26 (2018).

16. Seo, S. J., Kim, H. W. \& Lee, J. H. Electrospun nanofibers applications in dentistry. J. Nanomater. 2016, 1-7 (2016)

17. Zafar, M. et al. Potential of electrospun nanofibers for biomedical and dental applications. Materials 9, 1-21 (2016).

18. Monteiro, N. et al. Immobilization of bioactive factor-loaded liposomes on the surface of electrospun nanofibers targeting tissue engineering. Biomater. Sci. 2 1195-1209 (2014).

19. Jin, G. et al. Electrospun three-dimensional aligned nanofibrous scaffolds for tissue engineering. Mater. Sci. Eng. C. Mater. Biol. Appl 92, 995-1005 (2018).

20. Kang, D. H. \& Kang, H. W. Advanced electrospinning using circle electrodes for freestanding PVDF nanofiber film fabrication. Appl. Surf. Sci. 455, 251-257 (2018).

21. Cheng, J., Jun, Y., Qin, J. \& Lee, S. H. Electrospinning versus microfluidic spinning of functional fibers for biomedical applications. Biomaterials 114 121-143 (2017).

22. Wen, P., Zong, M.H., Linhardt, R. J., Feng, K. \& Wu, H. Electrospinning: a novel nano-encapsulation approach for bioactive compounds. Trend Food Sci. Tech. 70, 56-68 (2017).

23. Jiang, N., Sun, R. \& Sun, Q. Leptin signaling molecular actions and drug target in hepatocellular carcinoma. Drug Des. Devel. Ther. 8, 2295-2302 (2014).

24. de Candia, P. \& Matarese, G. Leptin and ghrelin: sewing metabolism onto neurodegeneration. Neuropharmacology 136, 307-316 (2018).

25. Zhang, W. F. et al. Protective effects of leptin against cerebral ischemia/ reperfusion injury. Exp. Ther. Med. 17, 3282-3290 (2019).

26. Guo, S. \& Gonzalez-Perez, R. R. Notch, IL-1 and leptin crosstalk outcome (NILCO) is critical for leptin-induced proliferation, migration and VEGFNEGFR-2 expression in breast cancer. PLOS ONE 6, 21467 (2011).

27. Selvaraj, S. \& Fathima, N. N. Fenugreek incorporated silkfibroin nanofibers-a potential antioxidant scaffold for enhanced wound healing. ACS Appl. Mater. Inter 9, 5916-5926 (2017).

28. Zhao, J. et al. Photothermal transforming agent and chemotherapeutic coloaded electrospun nanofibers for tumor treatment. Int. J. Nanomed. 14 3893-3909 (2019).

29. Zhang, Y. Z. et al. Coaxial electrospinning of (fluorescein isothiocyanateconjugated bovine serum albumin)-encapsulated poly(epsilon-caprolactone) nanofibers for sustained release. Biomacromolecules 7, 1049-1057 (2006).

30. Pant, B., Park, M. \& Park, S. J. Drug delivery applications of core-sheath nanofibers prepared by coaxial electrospinning: a review. Pharmaceutics $\mathbf{1 1}$, 1-21 (2019).

31. Xu, X. L. et al. Preparation of core-sheath composite nanofibers by emulsion electrospinning. Macromol. Rapid Commun. 27, 1637-1642 (2006).

32. Sun, Y. et al. Laden nanofiber capsules for local malignancy chemotherapy. J. Biomed. Nanotechnol. 15, 939-950 (2019).

33. Zhao, X. et al. Optimization of intrinsic and extrinsic tendon healing through controllable water-soluble mitomycin- $C$ release from electrospun fibers by mediating adhesion-related gene expression. Biomaterials 61, 61-74 (2015).

34. Miguel, S. P. et al. An overview of electrospun membranes loaded with bioactive molecules for improving the wound healing process. Eur. J. Pharm. Biopharm. 139, 1-22 (2019).

35. Liu, X. et al. An immunological electrospun scaffold for tumor cell killing and healthy tissue regeneration. Mater. Horiz. 5, 1082-1091 (2018).

36. Xiang, Y. et al. Self-nanoemulsifying electrospun fiber enhancing drug permeation. ACS Appl. Mater. Inter 11, 7836-7849 (2019).

37. Ge, Z., Yang, Q., Xiang, X. \& Liu, K. Z. Assessment of silk fibroin for the repair of buccal mucosa in a rat model. Int. J. Oral. Maxillofac. Surg. 41, 673-680 (2012). 
38. Lu, G. et al. Anisotropic biomimetic silk scaffolds for improved cell migration and healing of skin wounds. ACS Appl. Mater. Inter 10, 44314-44323 (2018).

39. Gou, S. et al. Multi-bioresponsive silk fibroin-based nanoparticles with ondemand cytoplasmic drug release capacity for CD44-targeted alleviation of ulcerative colitis. Biomaterials 212, 39-54 (2019).

40. Shen, Z., Chen, J., Kang, C., Gong, C. \& Zhu, Y. Engineered hypopharynx from coculture of epithelial cells and fibroblasts using poly(ester urethane) as substratum. Biomed. Res. Int. 2013, 138504 (2013).

41. $\mathrm{Tu}, \mathrm{H}$. et al. Layer-by-layer immobilization of amphoteric carboxymethyl chitosan onto biocompatible silk fibroin nanofibrous mats. Carbohydr. Polym. 210, 9-16 (2019).

42. Zafar, M. S., Belton, D. J., Hanby, B., Kaplan, D. L. \& Perry, C. C. Functional material features of Bombyx mori silk light versus heavy chain proteins. Biomacromolecules 16, 606-614 (2015).

43. Kim, S. H., Nam, Y. S., Lee, T. S. \& Park, W. H. Silk fibroin nanofiber: electrospinning, properties, and structure. Polym. J. 35, 185-190 (2003).

44. Bulbake, U., Doppalapudi, S., Kommineni, N. \& Khan, W. Liposomal formulations in clinical use: an updated review. Pharmaceutics 9, 1-33 (2017).

45. Cheng, R. et al. Mechanically enhanced lipo-hydrogel with controlled release of multi-type drugs for bone regeneration. Appl. Mater. Today 12, 294-308 (2018).

46. Liu, Y., Ai, K. \& Lu, L. Polydopamine and its derivative materials: synthesis and promising applications in energy, environmental, and biomedical fields. Chem. Rev. 114, 5057-5115 (2014).

47. Marelli, B. et al. Compliant electrospun silk fibroin tubes for small vessel bypass grafting. Acta Biomater. 6, 4019-4026 (2010)

48. Jones, G. L., Motta, A., Marshall, M. J., El Haj, A. J. \& Cartmell, S. H. Osteoblast: osteoclast co-cultures on silk fibroin, chitosan and PLLA films. Biomaterials $\mathbf{3 0}$, 5376-5384 (2009).

49. Velnar, T. \& Gradisnik, L. Tissue augmentation in wound healing: the role of endothelial and epithelial cells. Med. Arch. 72, 444-448 (2018).
50. Kenar, $H$. et al. Microfibrous scaffolds from poly(l-lactide-co-epsilon-caprolactone) blended with xeno-free collagen/hyaluronic acid for improvement of vascularization in tissue engineering applications. Mater. Sci. Eng. C. Mater. Biol. Appl. 97, 31-44 (2019).

51. Jin, L. et al. Three-dimensional nanofibrous microenvironment designed for the regulation of mesenchymal stem cells. Appl. Nanosci. 8, 1915-1924 (2018).

52. Zhang, Y. et al. An electrospun fiber-covered stent with programmable dual drug release for endothelialization acceleration and lumen stenosis prevention. Acta Biomater. 94, 295-305 (2019).

53. Liu, $H$. et al. Icariin immobilized electrospinning poly(l-lactide) fibrous membranes via polydopamine adhesive coating with enhanced cytocompatibility and osteogenic activity. Mater. Sci. Eng. C. Mater. Biol. Appl. 79, 399-409 (2017)

54. Zhao, J., Wang, X., Liu, L., Yu, J. \& Ding, B. Human skin-like, robust waterproof, and highly breathable fibrous membranes with short perfluorobutyl chains for eco-friendly protective textiles. ACS Appl. Mater. Interfaces 10, 30887-30894 (2018).

55. Kamalathevan, P., Ooi, P. S. \& Loo, Y. L. Silk-based biomaterials in cutaneous wound healing: a systematic review. Adv. Ski. Wound Care. 31, 565-573 (2018).

56. Yu, F. et al. Leptin-induced angiogenesis of EA.Hy926 endothelial cells via the Akt and Wht signaling pathways in vitro and in vivo. Front. Pharmacol. 10, 1275 (2019).

57. Cai, X. et al. Hemocompatibility improvement of poly(ethylene terephthalate) via self-polymerization of dopamine and covalent graft of zwitterions. Mater. Sci. Eng. C. Mater. Biol. Appl. 36, 42-48 (2014).

58. Crivelli, B. et al. Silk fibroin nanoparticles for celecoxib and curcumin delivery: ROS-scavenging and anti-inflammatory activities in an in vitro model of osteoarthritis. Eur. J. Pharm. Biopharm. 137, 37-45 (2019).

59. Wani, S. U. D. \& Veerabhadrappa, G. H. Silk fibroin based drug delivery applications: promises and challenges. Curr. Drug Targets 19, 1177-1190 (2018). 